\title{
What if gravity becomes really repulsive in the future?
}

\author{
Imanol Albarran ${ }^{1,2, a} \mathbb{C}$, Mariam Bouhmadi-López ${ }^{3,4, b}{ }_{\mathbb{C}}$, João Morais $^{3, c} \mathbb{C}_{\mathbb{C}}$ \\ ${ }^{1}$ Departamento de Física, Universidade da Beira Interior, Rua Marquês D’Ávila e Bolama, 6200-001 Covilhã, Portugal \\ 2 Centro de Matemática e Aplicações da Universidade da Beira Interior, Rua Marquês D’Ávila e Bolama, 6200-001 Covilhã, Portugal \\ ${ }^{3}$ Department of Theoretical Physics, University of the Basque Country UPV/EHU, P.O. Box 644, 48080 Bilbao, Spain \\ ${ }^{4}$ IKERBASQUE, Basque Foundation for Science, 48011 Bilbao, Spain
}

Received: 2 August 2017 / Accepted: 10 March 2018 / Published online: 24 March 2018

(C) The Author(s) 2018

\begin{abstract}
The current acceleration of the Universe is one of the most puzzling issues in theoretical physics nowadays. We are far from giving an answer in this letter to the question of its nature. Yet, with the observations we have at hand, we analyse the different patterns that the gravitational potential can show in the future. Surprisingly, gravity not only can get weaker in the near future, it can even become repulsive; or equivalently, the gravitational potential may become negative. We show this remark by using one of the simplest phenomenological model we can imagine for dark energy. We have also reviewed the statefinder approach of these models. For completeness, we have also showed the behaviour of the density contrast of dark matter and dark energy for these simple (yet illustrative models). Our results are displayed and we see how they shall evolve in the future.
\end{abstract}

\section{Introduction}

Hubble's discovery was crucial for our understanding of the Universe. He showed that the Universe was evolving and not static as it was believed at that time [1]. His discovery was based on observing that the spectrum of far away galaxies was red-shifted which implied that those galaxies were moving away from us. He even measured the galaxies radial outward velocities and realised that it followed a rule: (1) the velocities were proportional to the distances at which the galaxies were located from us and (2) the proportionality factor was a constant, the Hubble constant. About 70 years later, two independent teams $[2,3]$ realised that by measuring further objects, SNeIa, the Hubble constant was not quite constant, as was already expected. The issue was that the deviation from the constancy was not in the anticipated direction. It

\footnotetext{
a e-mail: albarran.payo@ubi.pt

b e-mail: mariam.bouhmadi@ehu.eus

c e-mail: jviegas001@ikasle.ehu.eus
}

was no longer enough to invoke only matter to explain those observations. A new dark component had to be invoked, interacting as far as we know only gravitationally, and named dark energy. This component started recently fuelling a second inflationary era of the visible Universe. Of course, all these observations, and subsequent ones, are telling us how gravity behaves at cosmological scales through the kinematic expansion of our Universe [4-9].

This kinematic description is linked to the dynamical expansion through the gravitational laws of Einstein theory. To a very good approximation, we may assume that our Universe is homogeneous and isotropic on large scales and that it is filled with matter (standard and dark) and dark energy, where their relative fractional energy densities are $\Omega_{\mathrm{m}}=0.309$ and $\Omega_{\mathrm{d}}=0.691$, respectively, at present. In addition, the current Hubble parameter is of the order of $H_{0}=67.74 \mathrm{~km} \mathrm{~s}^{-1} \mathrm{Mpc}^{-1}$. We have fixed those values by using the latest Planck data [7] but please notice that our conclusions in this paper are unaltered by choosing other values for these physical quantities. As regards dark energy, we will assume its energy density to be evolving (or not) in time and its equation of state $(\mathrm{EoS})$ parameter, $w$, to be constant; i.e. we will consider the $w \mathrm{CDM}$ model as a natural candidate describing our Universe. As is well known: (1) for $w<-1$ the Universe would face a big rip singularity [10-12], i.e., the Universe would unzip itself in a finite time from now, (2) for $w=-1$ the Universe would be asymptotically de Sitter, and finally (3) if $w>-1$ the Universe would be asymptotically flat locally; i.e. the scalar curvature and the Ricci tensor would vanish for large scale factors. As we next show this pattern is shown also by the behaviour of the gravitational potential.

The paper is organised as follows: in Sect. 2, we review briefly the models to be considered and compare them using a cosmographic/statefinder analysis. In Sect. 3, we present the cosmological perturbations of the models focussing on the 
asymptotic behaviour of the gravitational potential. Finally, in Sect. 4, we conclude. In Appendix A, we include some formulae useful in Sect. 2.

\section{Background approach}

The geometry of the cosmological background is adequately given by the Friedmann-Lemaître-Robertson-Walker line element:

$\mathrm{d} s^{2}=-\mathrm{d} t^{2}+a^{2} \delta_{i j} \mathrm{~d} x^{i} \mathrm{~d} x^{j}$,

where $t$ is the cosmic time, $a(t)$ is the scale factor and $\delta_{i j}$ is the flat spatial metric. On the other hand, the matter content of the Universe can be separated in three main components: radiation, nonrelativistic matter (baryons and dark matter (DM)) and dark energy (DE). For simplicity, we model these three components using a perfect fluid description where each fluid has energy density $\rho_{\mathrm{i}}$ and pressure $p_{\mathrm{i}}=w_{\mathrm{i}} \rho_{\mathrm{i}}$. Here, i stands for radiation ( $\mathrm{r}$ ) with $w_{\mathrm{r}}=1 / 3$, for nonrelativistic matter $(\mathrm{m})$ with $w_{\mathrm{m}}=0$, and for DE (d) with $w_{\mathrm{d}}=w$. The Friedmann equation for such model can be written as

$$
\frac{H^{2}}{H_{0}^{2}}=\Omega_{\mathrm{r}, 0}\left(\frac{a_{0}}{a}\right)^{4}+\Omega_{\mathrm{m}, 0}\left(\frac{a_{0}}{a}\right)^{3}+\Omega_{\mathrm{d}, 0}\left(\frac{a_{0}}{a}\right)^{3(1+w)}
$$

where the various $\Omega_{\mathrm{i}, 0}:=\kappa^{2} \rho_{\mathrm{i}, 0} /\left(3 H_{0}^{2}\right)$ represent the present day fractional energy density of the different fluids and satisfy the constraint $1=\Omega_{\mathrm{r}, 0}+\Omega_{\mathrm{m}, 0}+\Omega_{\mathrm{d}, 0}$. In this work, we adopt three different values for $w:\{-0.99,-1$, $-1.01\}$, in order to obtain three qualitatively different types of late-time behaviour for DE: quintessence $(w \gtrsim-1)$, cosmological constant $(w=-1)$ and phantom behaviour $(w \lesssim-1)$.

In a cosmographic approach [13-16], the scale factor is Taylor expanded around its present day value $a_{0}:=a\left(t_{0}\right)$ as

$$
\frac{a(t)}{a_{0}}=1+\sum_{n=1}^{\infty} \frac{A_{n}\left(t_{0}\right)}{n !}\left[H_{0}\left(t-t_{0}\right)\right]^{n} .
$$

Here, $H_{0}$ is the present day value of the Hubble rate $H:=$ $\dot{a} / a$, where a dot represents a derivative with respect to the cosmic time, and the cosmographic parameters $A_{n}$ are defined as $A_{n}:=a^{(n)} /\left(a H^{n}\right), n \in \mathbb{N}$, where $a^{(n)}$ is the $n$th derivative of the scale factor with respect to the cosmic time. ${ }^{1}$ Based on the cosmographic expansion (3), the

\footnotetext{
1 The parameters $A_{2}, A_{3}, A_{4}, A_{5}$ are also known as the deceleration parameter $q=-A_{2}$, the jerk $j=A_{3}$, the snap $s=A_{4}$ and the lerk $l=A_{5}$, respectively [13].
}

statefinder hierarchy was developed as a tool to distinguish different DE models [17-21]. In fact, the statefinder parameters are defined as specific combinations of the cosmographic parameters:

$S_{3}^{(1)}=A_{3}$,

$S_{4}^{(1)}=A_{4}+3\left(1-A_{2}\right)$,

$S_{5}^{(1)}=A_{5}-2\left(4-3 A_{2}\right)\left(1-A_{2}\right)$,

such that, by construction, $\left.S_{n}^{(1)}\right|_{\Lambda \mathrm{CDM}}=1$, i.e., the statefinder hierarchy defines a null diagnostic for the $\Lambda \mathrm{CDM}$ model [20]. It is also convenient to introduce the statefinder parameter $s$ defined in $[17,18]$ as

$s=\frac{1-S_{3}^{(1)}}{3\left(A_{2}+\frac{1}{2}\right)}$.

For the case of a $w \mathrm{CDM}$ model with a radiation component, such as the models considered in this paper, we present in Appendix A the full expressions of the statefinder parameters as functions of the scale factor $a / a_{0}$ and the cosmological parameters $\left\{\Omega_{\mathrm{i}, 0}, w\right\}$. In the limit $a \rightarrow+\infty$ the expressions found reduce to

$$
\begin{aligned}
\left.S_{3}^{(1)}\right|_{w \mathrm{CDM}} & =1+\frac{9}{2} w(1+w), \\
\left.S_{4}^{(1)}\right|_{w \mathrm{CDM}} & =1-\frac{9}{4} w(1+w)(7+9 w), \\
\left.S_{5}^{(1)}\right|_{w \mathrm{CDM}} & =1+\frac{9}{4} w(1+w)\left(41+87 w+54 w^{2}\right), \\
\left.s\right|_{w \mathrm{CDM}} & =1+w .
\end{aligned}
$$

We thus find that as $w$ deviates from the nominal value -1 the asymptotic values of the statefinder parameters $S_{i}^{(1)}$ run away from unity. In fact, for small deviations $\Delta w:=|w+1| \ll 1$ the statefinder parameters depend linearly on $\Delta w$ and we find that $S_{n}^{(1)}<1$ for quintessence models and $S_{n}^{(1)}>1$ in the case of phantom behaviour. On the other hand, it can be shown that asymptotically $s$ vanishes for $\Lambda \mathrm{CDM}$, and it gets negative for $w<-1$ and positive for $-1<w$. We have assumed in all our conclusions the presence of radiation no matter how tiny its contribution.

On Fig. 1, we present the evolution of the statefinder hierarchy $\left\{S_{3}^{(1)}, s\right\}$ (top panel), $\left\{S_{3}^{(1)}, S_{4}^{(1)}\right\}$ (middle panel) and $\left\{S_{3}^{(1)}, S_{5}^{(1)}\right\}$ (bottom panel) for the three models considered: $w=-0.99$ (blue), $w=-1$ (green) and $w=-1.01$ (red). When the Universe is dominated by radiation and matter the three models are indistinguishable and can be seen to follow the same straight line trajectory in the $\left\{S_{3}^{(1)}, s\right\},\left\{S_{3}^{(1)}, S_{4}^{(1)}\right\}$ and $\left\{S_{3}^{(1)}, S_{5}^{(1)}\right\}$ planes. However, as DE starts to dominate at late time the differences between the three models become apparent. The trajectory $\left\{S_{3}^{(1)}, s\right\}$ evolves towards the point 


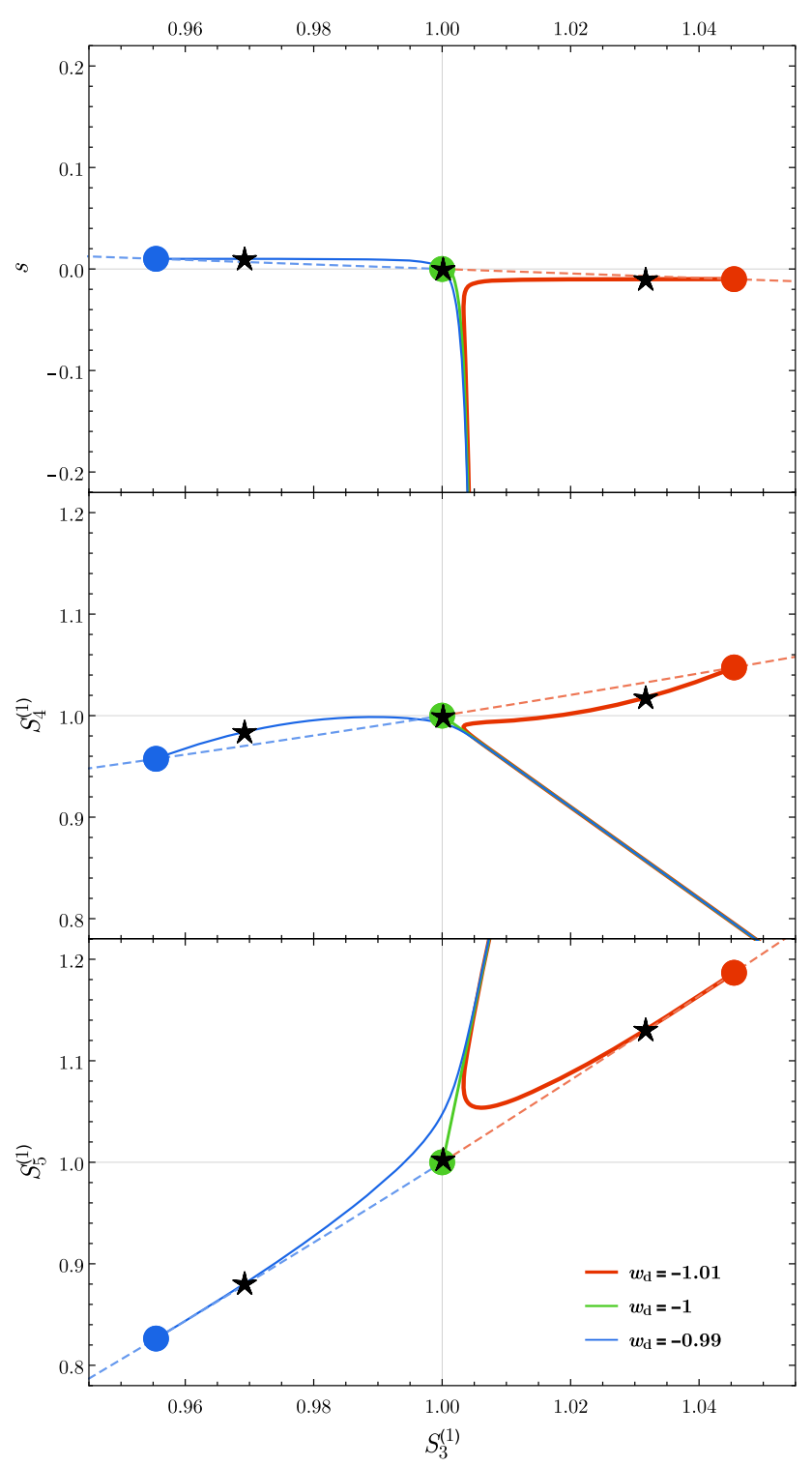

Fig. 1 This figure shows the trajectory of the three models considered in this work in the $\left\{S_{3}^{(1)}, s\right\},\left\{S_{3}^{(1)}, S_{4}^{(1)}\right\}$ and $\left\{S_{3}^{(1)}, S_{5}^{(1)}\right\}$ planes, which characterise the statefinder hierarchy. The coloured points indicate the asymptotic values of the statefinder parameters as presented in Eqs. (8)(10). The dependence of these points on the deviation of $w$ from the $\Lambda \mathrm{CDM}$ value -1 is illustrated by the dashed lines. The black stars indicate the present day values of the statefinder parameters for each of the models

$(1,0)$ for the $\Lambda \mathrm{CDM}$ model, then for a quintessence model that trajectory evolves towards the second quadrant in the plane $\left\{S_{3}^{(1)}, s\right\}$, i.e. $S_{3}^{(1)}<1$ and $0<s$, and, finally, for a phantom scenario the trajectory $\left\{S_{3}^{(1)}, s\right\}$ heads towards the fourth quadrant, i.e. $1<S_{3}^{(1)}$ and $s<0$. For the second group of trajectories, $\left(\left\{S_{3}^{(1)}, S_{4}^{(1)}\right\}\right.$ and $\left.\left\{S_{3}^{(1)}, S_{5}^{(1)}\right\}\right)$, the trajectories of the model with $w=-1$ evolve towards the point $(1,1)$ that characterises $\Lambda \mathrm{CDM}$, in the quintessence model the trajectories evolve towards the third quadrant in both pan- els $\left(S_{n}^{(1)}<1\right.$ for $\left.n=3,4,5\right)$. In contrast, for the model with phantom behaviour the trajectories evolve towards the first quadrant in the $\left\{S_{3}^{(1)}, S_{4}^{(1)}\right\}$ and $\left\{S_{3}^{(1)}, S_{5}^{(1)}\right\}$ planes characterised by $S_{n}^{(1)}>1$ for $n=3,4,5$. Finally, by looking at Fig. 1, it seems that the pair $\left\{S_{3}^{(1)}, S_{5}^{(1)}\right\}$ are suitable to distinguish the model with $w<-1$ from $-1<w$.

\section{Cosmological perturbations: from gravity to DM and DE}

The gravitational potential can be described through the time-time metric component as

$\mathrm{d} s^{2}=a^{2}\left[-(1+2 \Phi) \mathrm{d} \eta^{2}+(1-2 \Phi) \delta_{i j} \mathrm{~d} x^{i} \mathrm{~d} x^{j}\right]$,

where $\eta$ is the conformal time, $\delta_{i j}$ is the flat spatial metric and $\Phi$ the gravitational potential. For simplicity, we assume the absence of anisotropies; i.e. the spatial and temporal component of the gravitational metric are equal in absolute values at first order in the cosmological perturbations.

In order to tackle the cosmological perturbations of a perfect fluid with a negative and constant EoS some care has to be taken into account [22]. In fact, unless non-adiabatic perturbations are taken into account a blow up on the cosmological perturbations quickly appears even at scales we have already observed. Please notice that this is so even for non-phantom fluids, i.e., for $w \geq-1$. This will be our first assumption and therefore non-adiabatic perturbations will be considered. The non-adiabaticity implies the existence of two distinctive speed of sounds for the dark energy fluid: (1) its quadratic adiabatic speed of sound $c_{a}^{2}=w$ (in our case) and (2) its effective quadratic speed of sound, $c_{s}^{2}$, whose deviation from $c_{a}^{2}=w$ measures the non-adiabaticity in the evolution of the fluid [23]. For simplicity, we will set the latter to one which fits perfectly the case of a scalar field, no matter if it is a canonical scalar field of standard or phantom nature. ${ }^{2}$ In addition, we will solve the gravitational equations describing the cosmological perturbations at first order using the same methodology we presented in [22]. We remind the reader that the temporal and spatial components of the conservation equation of each fluid imply [22]

$$
\delta_{\mathrm{r}}^{\prime}=4\left(\frac{k^{2}}{3} v_{\mathrm{r}}+\Phi^{\prime}\right)
$$

\footnotetext{
${ }^{2}$ As long as the speed of sound $c_{s}^{2}$ is not too close to zero and $w \simeq-1$, the value of $c_{s}^{2}$ will not much affect the perturbations of dark matter. A full discussion on the effect of the speed of sound of DE on the perturbations of the late Universe can be found in [24-26]. Therefore, our choice $c_{s}^{2}=1$ is not crucial in our study, it was taken just for simplicity and because it is common to use it in codes like CAMB and CLASS, though there is no fundamental reason for such a choice.
} 
$v_{\mathrm{r}}^{\prime}=-\left(\frac{1}{4} \delta_{\mathrm{r}}+\Phi\right)$

$\delta_{\mathrm{m}}^{\prime}=3\left(\frac{k^{2}}{3} v_{\mathrm{m}}+\Phi^{\prime}\right)$,

$v_{\mathrm{m}}^{\prime}=-\left(\mathscr{H} v_{\mathrm{m}}+\Phi\right)$,

$\delta_{\mathrm{d}}^{\prime}=3(w-1) \delta_{\mathrm{d}}$

$$
+3(1+w)\left\{\left[\frac{k^{2}}{3}+3 \mathscr{H}^{2}(1-w)\right] v_{\mathrm{d}}+\Phi^{\prime}\right\},
$$

$v_{\mathrm{d}}^{\prime}=-\left(\frac{1}{1+w} \delta_{\mathrm{d}}+\Phi\right)+2 \mathscr{H} v_{\mathrm{d}}$

while the $(00)$ and $(0 i)$ components of the Einstein equations lead to [22]

$$
\begin{aligned}
\mathscr{H} \Phi^{\prime}+\left(\mathscr{H}^{2}+\frac{k^{2}}{3}\right) \Phi & =-\frac{1}{2} \mathscr{H}^{2} \delta_{\text {tot }}, \\
\Phi^{\prime}+\mathscr{H} \Phi & =-\frac{3}{2} \mathscr{H}^{2}\left(1+w_{\text {tot }}\right) v_{\text {tot }} .
\end{aligned}
$$

In the previous equations, $\mathscr{H}:=a^{\prime} / a$ is the conformal Hubble rate, $\delta_{i}$ and $v_{i}$ correspond to the density contrast and peculiar velocity of the fluid $i$, and we have decomposed all the perturbations into their Fourier modes. The total quantities $w_{\text {tot }}, \delta_{\text {tot }}$ and $v_{\text {tot }}$ found in (19) and (20) are defined through a proper averaging of the individual fluid values:

$w_{\text {tot }}=\frac{\sum_{\mathrm{i}=\mathrm{r}, \mathrm{m}, \mathrm{d}} \rho_{\mathrm{i}} w_{\mathrm{i}}}{\sum_{\mathrm{i}=\mathrm{r}, \mathrm{m}, \mathrm{d}} \rho_{\mathrm{i}}}$,

$\delta_{\mathrm{tot}}=\frac{\sum_{\mathrm{i}=\mathrm{r}, \mathrm{m}, \mathrm{d}} \rho_{\mathrm{i}} \delta_{\mathrm{i}}}{\sum_{\mathrm{i}=\mathrm{r}, \mathrm{m}, \mathrm{d}} \rho_{\mathrm{i}}}$,

$v_{\text {tot }}=\frac{\sum_{\mathrm{i}=\mathrm{r}, \mathrm{m}, \mathrm{d}} \rho_{\mathrm{i}}\left(1+w_{\mathrm{i}}\right) v_{\mathrm{i}}}{\sum_{\mathrm{i}=\mathrm{r}, \mathrm{m}, \mathrm{d}} \rho_{\mathrm{i}}\left(1+w_{\mathrm{i}}\right)}$.

In order to integrate (13)-(18) [after assuming (19) and (20)] we impose the standard adiabatic initial conditions [22]

$\frac{3}{4} \delta_{\mathrm{r}, \text { ini }}=\delta_{\mathrm{m}, \text { ini }}=\frac{\delta_{\mathrm{d}, \mathrm{ini}}}{1+w} \approx \frac{3}{4} \delta_{\mathrm{tot}, \mathrm{ini}}$

and

$v_{\mathrm{r}, \text { ini }}=v_{\mathrm{m}, \mathrm{ini}}=v_{\mathrm{d}, \mathrm{ini}} \approx v_{\mathrm{tot}, \mathrm{ini}}$,

while Eqs. (19) and (20) imply

$\Psi_{\mathrm{ini}} \approx-\frac{1}{2} \delta_{\mathrm{tot}, \mathrm{ini}}$

$\Psi_{\text {ini }} \approx-2 \mathscr{H}_{\text {ini }} v_{\text {tot,ini }}$

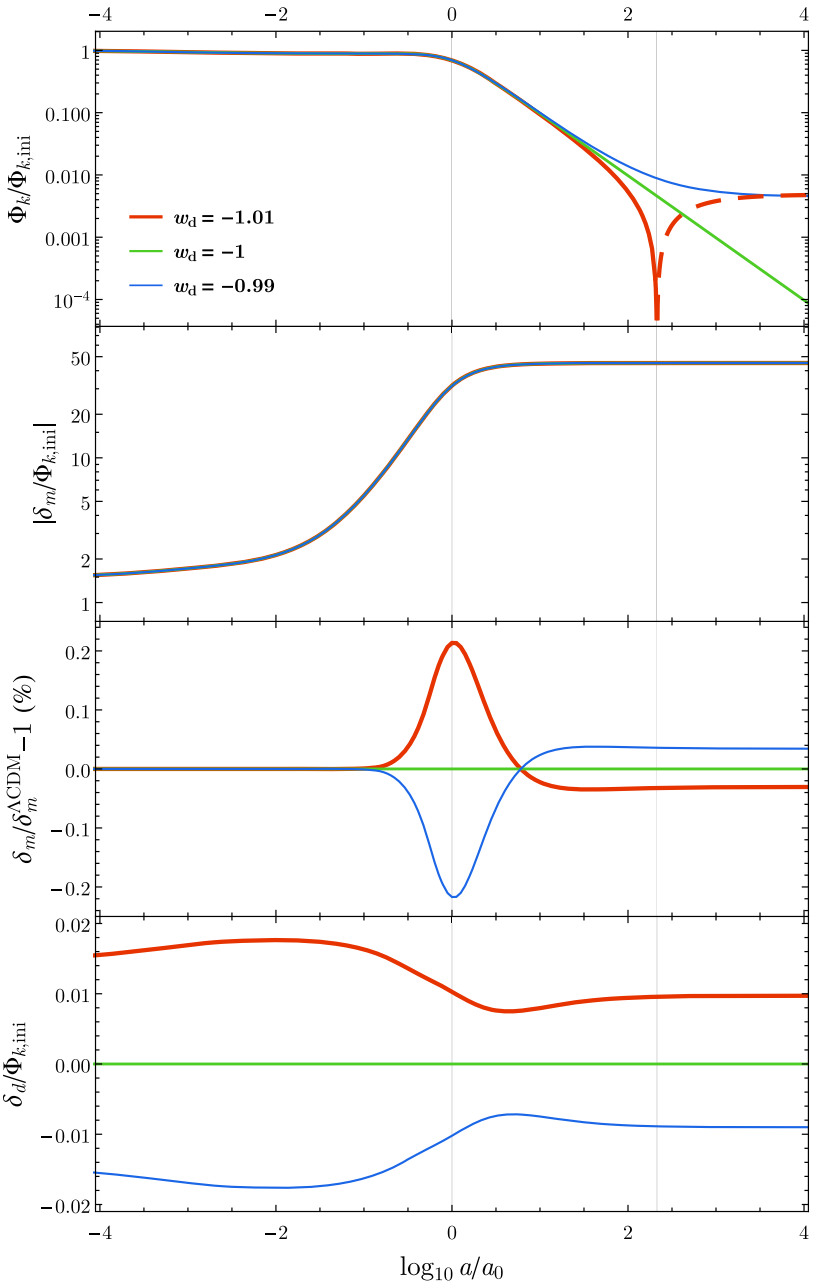

Fig. 2 The evolution of the Fourier mode of the gravitational potential $\Phi_{k}$ (top panel), the DM perturbation $\delta_{\mathrm{m}}$ (middle panels) and the DE perturbation (bottom panel), from the matter era to the far future for the mode $k=10^{-3} \mathrm{Mpc}^{-1}$ and for three dark energy models: (blue) $w=-0.99$, (green) $w=-1$ and (red) $w=-1.01$. For the quintessence model (blue) the gravitational potential evolves towards a constant in the far future without changing sign, while for $\Lambda \mathrm{CDM}$ (green) $\Phi_{k}$ vanishes asymptotically. In the phantom model (red), $\Phi_{k}$ also evolves towards a constant in the far future but a change of sign occurs roughly at $\log _{10} a / a_{0} \simeq 2.33$, corresponding to $8.84 \times 10^{10}$ years in the future. A dashed line indicates negative values of $\Phi_{k}$

These initial conditions are fully fixed by the Planck observational fit to single inflation [7]:

$\Phi_{\text {ini }}=\frac{2 \pi}{3} \sqrt{2 A_{s}}\left(\frac{k}{k_{*}}\right)^{n_{s}-1} k^{-3 / 2}$,

where $A_{s}=2.142 \times 10^{-9}, n_{s}=0.9667$ and the pivot scale is $k_{*}=0.05 \mathrm{Mpc}^{-1}$.

The behaviour of the gravitational potential and the perturbations is shown in the top panel of Fig. 2 for a given scale. We choose as an example $k=10^{-3} \mathrm{Mpc}^{-1}$. As it must, the gravitational potential is constant during the matter era and 
starts decreasing as soon as dark energy goes on stage. This behaviour is independent of the dark energy model considered. However, shortly afterwards, i.e., in our near future, the gravitational potential will depend on the specifically chosen EoS for dark energy. In fact: (1) it will decrease until reaching a positive non-vanishing value at infinity for $w>-1$, (2) it will vanish asymptotically for $w=-1$, and amazingly (3) it will vanish and become negative for $w<-1$ ! This is in full agreement with the fact that close to the big rip the different structures in our Universe will be destroyed no matter their sizes or bounding energies. When could the gravitational potential vanish and flip its sign? Of course, the answer is model and scale dependent [22]. For the model we have considered, the gravitational potential for the mode $k=10^{-3} \mathrm{Mpc}^{-1}$ will vanish in $8.84 \times 10^{10}$ years from the present time or equivalently when the Universe is roughly 213 times its current size. Furthermore, numerical results show that the smaller the scale that is considered (larger $k$ ), the later the gravitational potential will flip sign [22].

In addition to the gravitational potential, we present in the second and third panels of Fig. 2 the behaviour of the density contrast of DM. We observe that the growth of the linear perturbations is very similar in all models, with differences of $\lesssim 0.2 \%$ with regards to $\Lambda \mathrm{CDM}$. However, when comparing the phantom DE model with $\Lambda \mathrm{CDM}$ we find that until the present time there is an excess in the growth of the linear perturbations of DM in the phantom DE case. In the case of quintessence the opposite behaviour is observed: until the present time $\delta_{\mathrm{m}}$ is smaller in the quintessence case when compared with $\Lambda \mathrm{CDM}$. This effect, which depends on the qualitative behaviour of DE, was first noted in [10]. Surprisingly, these deviations peak around the present time and their sign reverses in the near future. On the bottom panel of Fig. 2 we present the evolution of $\delta_{\mathrm{DE}}$ for the different models. Of course, for the $\Lambda \mathrm{CDM}$ case the perturbations remain at 0 as the cosmological constant does not cluster. In good agreement with observations, for the quintessence and phantom DE models we find that the DE perturbations remain small, with small variations of the initial value, throughout the whole evolution of the Universe.

Finally, and most importantly, all these models are in full agreement with observations. In Fig. 3, we show the evolution of the observable $f \sigma_{8}$ for the three models mentioned above. This combination of $f$, the relative growth of the linear matter perturbations, and $\sigma_{8}$, the root-mean-square mass fluctuation in spheres with radius $8 \mathrm{~h}^{-1} \mathrm{Mpc}$, was proposed in [27] as a discriminant for different models of late-time acceleration that is independent of local galaxy density bias. On the top panel of Fig. 3, we contrast the $f \sigma_{8}$ curves of the three models with the available observational data (cf. Table I of [22]). All the three curves, which are practically indistinguishable for the naked eye, are within the error bars of nearly all the points. On the bottom panel of Fig. 3, we present the

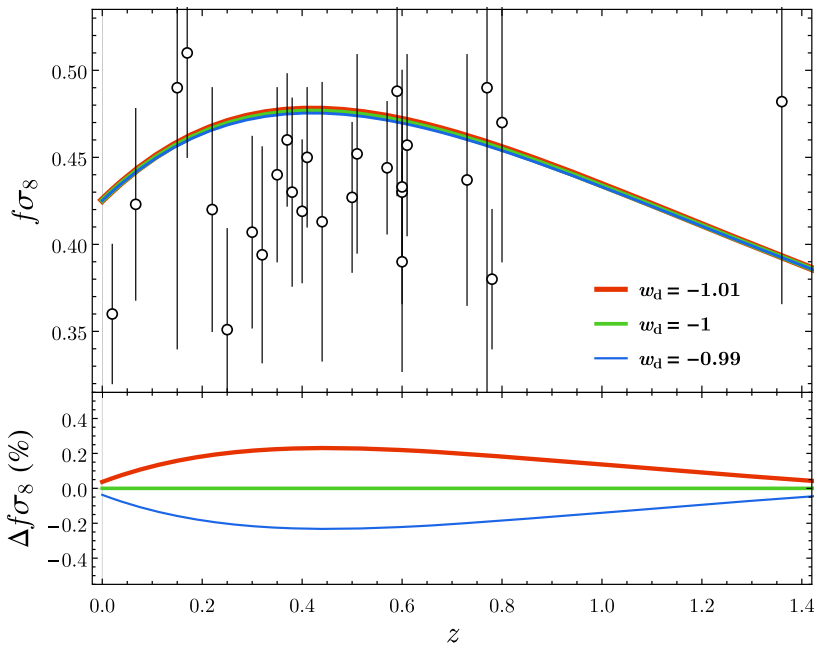

Fig. 3 (Top panel) evolution of $f \sigma_{8}$ for low red-shift $z \in(0,1.4)$ for three dark energy models: (blue) $w=-0.99$, (green) $w=-1$ and (red) $w=-1.01$. White circles and vertical bars indicate the available data points and corresponding error bars (cf. Table I of [22]). (Bottom panel) Evolution of the relative differences of $f \sigma_{8}$ for each model with regard to $\Lambda \mathrm{CDM}(w=-1) . \Delta f \sigma_{8}$ is positive in the phantom case and negative in the quintessence case. For all the models, it was considered that $\sigma_{8}$ evolves linearly with $\delta_{\mathrm{m}}$ and that $\sigma_{8}=0.816$ at the present time [7]

relative difference, $\Delta f \sigma_{8}$, of the results of each model with regards to $\Lambda \mathrm{CDM} .^{3}$ Despite the small values found in terms of amplitudes, the behaviour observed suggests that the sign of $\Delta f \sigma_{8}$ can distinguish between a phantom (positive $\Delta f \sigma_{8}$ ) and a quintessence model (negative $\Delta f \sigma_{8}$ ). As a consequence of this difference in sign, the growth of the linear matter perturbations is stronger in a phantom scenario as opposed to $\Lambda \mathrm{CDM}$ and quintessence. This is in full agreement with the results presented in [10] where the decay of the growth suppression factor of the linear matter perturbations is found to be faster in quintessence models and slower in phantom models.

\section{Concluding remarks}

Summarising, what we have shown is that after all gravity might behave the other way around in the future and, rather than the apple falling from the tree, the apple may fly from the earth surface to the branches of the tree, if dark energy is repulsive enough, as could already be indicated by current observations. $^{4}$

\footnotetext{
$\overline{3 \Delta f \sigma_{8}^{(\text {model })}(\%)}:=100\left[\left(f \sigma_{8}^{(\text {model })}\right) /\left(f \sigma_{8}^{\Lambda \mathrm{CDM}}\right)-1\right]$.

${ }^{4}$ Repulsive gravity could occur as well if the effective gravitational constant changes sign. This could happen, for example, in scalar-tensor theories, in particular, for a non-minimally coupled scalar field [28]. However, an anisotropic curvature singularity arises generically at the moment of this transition.
} 
To illustrate these observations, we have considered three models where DE is characterised by a constant parameter of $\operatorname{EoS} w$ with values $w=-0.99,-1,-1.01$. After comparing the present and future behaviour at the background level by using a statefinder approach, as illustrated in Fig. 1, we have considered the cosmological perturbations of these models. We have shown that for models with $w<-1$ the gravitational potential changes sign in the future (cf. Fig. 2). We have as well analysed the behaviour of the DM and DE perturbations as shown for example in Fig. 2. Finally, we have proven that no matter the future behaviour of the gravitational potential depicted in Fig. 2, the three models discussed above are in full agreement with the latest observations of $f \sigma_{8}$ (cf. Fig. 3).

Before concluding, we would like to recall that in this work, we have considered the existence of phantom matter; however, it might be possible that Nature presents rather a phantom-like behaviour as happens in brane world-models $[29,30]$ where no big rip takes place and where the perturbations can be stable. In addition, even the presence of phantom matter might not be a problem at a cosmological quantum level where the big rip or other kind of singularities can be washed away [31-33].

Acknowledgements The work of IA was supported by a SantanderTotta fellowship "Bolsas de Investigação Faculdade de Ciências (UBI) Santander Totta". The work of MBL is supported by the Basque Foundation of Science IKERBASQUE. JM is thankful to UPV/EHU for a $\mathrm{PhD}$ fellowship. MBL and JM acknowledge financial support from project FIS2017-85076-P (MINECO/AEI/FEDER, UE), and Basque Government Grant no. IT956-16. This research work is supported by the Grant UID/MAT/00212/2013. This paper is based upon work from COST action CA15117 (CANTATA), supported by COST (European Cooperation in Science and Technology).

Open Access This article is distributed under the terms of the Creative Commons Attribution 4.0 International License (http://creativecomm ons.org/licenses/by/4.0/), which permits unrestricted use, distribution, and reproduction in any medium, provided you give appropriate credit to the original author(s) and the source, provide a link to the Creative Commons license, and indicate if changes were made. Funded by SCOAP ${ }^{3}$.

\section{Appendix A: Statefinder parameters in $w \mathrm{CDM}$}

For a $w$ CDM model with a radiation component the statefinder parameters defined in Eqs. (4), (5), (6) and (7) read

$$
\begin{aligned}
S_{3}^{(1)}= & 1+\frac{2 \Omega_{\mathrm{r}, 0} \frac{a_{0}}{a}+\frac{9}{2} w(1+w) \Omega_{\mathrm{d}, 0}\left(\frac{a_{0}}{a}\right)^{3 w}}{\Omega_{\mathrm{r}, 0} \frac{a_{0}}{a}+\Omega_{\mathrm{m}, 0}+\Omega_{\mathrm{d}, 0}\left(\frac{a_{0}}{a}\right)^{3 w}}, \\
S_{4}^{(1)}= & 1-\left[\Omega_{\mathrm{r}, 0} \frac{a_{0}}{a}+\Omega_{\mathrm{m}, 0}+\Omega_{\mathrm{d}, 0}\left(\frac{a_{0}}{a}\right)^{3 w}\right]^{-2} \\
\times & \left\{\left[10 \Omega_{\mathrm{r}, 0} \frac{a_{0}}{a}\right.\right.
\end{aligned}
$$

$$
\begin{aligned}
+ & 9 \Omega_{\mathrm{m}, 0}+\left(9+\frac{3}{2} w(14+3 w(7+3 w))\right) \\
& \left.\times \Omega_{\mathrm{d}, 0}\left(\frac{a_{0}}{a}\right)^{3 w}\right]+\frac{9}{4} w(1+w) \\
& \times\left[(7+6 w) \Omega_{\mathrm{m}, 0}+(7+9 w) \Omega_{\mathrm{d}, 0}\left(\frac{a_{0}}{a}\right)^{3 w}\right] \\
& \left.\times \Omega_{\mathrm{d}, 0}\left(\frac{a_{0}}{a}\right)^{3 w}\right\}, \\
S_{5}^{(1)}= & 1+\left[\Omega_{\mathrm{r}, 0} \frac{a_{0}}{a}+\Omega_{\mathrm{m}, 0}+\Omega_{\mathrm{d}, 0}\left(\frac{a_{0}}{a}\right)^{3 w}\right]^{-2}\left\{\left[76 \Omega_{\mathrm{r}, 0} \frac{a_{0}}{a}\right.\right. \\
& +60 \Omega_{\mathrm{m}, 0}+\left(60+\frac{3}{2} w\left(37+w\left(59+39 w+9 w^{2}\right)\right)\right) \\
& \left.\times \Omega_{\mathrm{d}, 0}\left(\frac{a_{0}}{a}\right)^{3 w}\right] \Omega_{\mathrm{r}, 0} \frac{a_{0}}{a}+\frac{9}{4} w(1+w) \\
& \times\left[(41+3 w(17+6 w)) \Omega_{\mathrm{m}, 0}+\left(41+87 w+54 w^{2}\right)\right. \\
& \left.\left.\times \Omega_{\mathrm{d}, 0}\left(\frac{a_{0}}{a}\right)^{3 w}\right] \Omega_{\mathrm{d}, 0}\left(\frac{a_{0}}{a}\right)^{3 w}\right\} . \\
s & \frac{4 \Omega_{\mathrm{r}, 0}}{3} \frac{a_{0}}{a}+9 w(1+w) \Omega_{\mathrm{d}, 0}\left(\frac{a_{0}}{a}\right)^{3 w} \\
& 3 \Omega_{\mathrm{r}, 0} \frac{a_{0}}{a}+9 w \Omega_{\mathrm{d}, 0}\left(\frac{a_{0}}{a}\right)^{3 w}
\end{aligned}
$$

Due to the Friedmann constraint $1=\Omega_{\mathrm{r}, 0}+\Omega_{\mathrm{m}, 0}+\Omega_{\mathrm{d}, 0}$ we can eliminate one of the fractional energy density parameters. It can be checked that, for the $\Lambda \mathrm{CDM}$ model, where $\Omega_{\mathrm{r}, 0}=0$ and $w=-1$, the previous expressions reduce to $S_{n}^{(1)}=1$ and $s=0$.

\section{References}

1. E. Hubble, Proc. Nat. Acad. Sci. 15, 168 (1929)

2. A.G. Riess et al. [Supernova Search Team], Astron. J 116, 1009 (1998)

3. S. Perlmutter et al. [Supernova Cosmology Project Collaboration], Bull. Am. Astron. Soc. 29, 1351 (1997)

4. W.J. Percival et al. [2dFGRS Collaboration], Mon. Not. R. Astron. Soc. 353, 1201 (2004)

5. C. Blake et al., Mon. Not. R. Astron. Soc. 415, 2876 (2011)

6. F. Beutler et al., Mon. Not. R. Astron. Soc. 423, 3430 (2012)

7. P.A.R. Ade et al. [Planck Collaboration], Astron. Astrophys. 594, A13 (2016)

8. T. Abbott et al. [DES Collaboration], Mon. Not. R. Astron. Soc. 460, 1270 (2016)

9. S. Satpathy et al. [BOSS Collaboration], Mon. Not. R. Astron. Soc. 469, 1369 (2017)

10. R.R. Caldwell, Phys. Lett. B 545, 23 (2002)

11. A.A. Starobinsky, Grav. Cosmol. 6, 157 (2000)

12. R.R. Caldwell, M. Kamionkowski, N.N. Weinberg, Phys. Rev. Lett. 91, 071301 (2003)

13. M. Visser, Gen. Rel. Grav. 37, 1541 (2005)

14. C. Cattoen, M. Visser, Class. Quant. Grav. 24, 5985 (2007)

15. S. Capozziello, V.F. Cardone, V. Salzano, Phys. Rev. D 78, 063504 (2008)

16. J. Morais, M. Bouhmadi-López, S. Capozziello, JCAP 1509(09), 041 (2015) 
17. V. Sahni, T.D. Saini, A.A. Starobinsky, U. Alam, JETP Lett. 77, $201(2003)$

18. V. Sahni, T.D. Saini, A.A. Starobinsky, U. Alam, Pisma. Zh. Eksp. Teor. Fiz. 77, 249 (2003)

19. U. Alam, V. Sahni, T.D. Saini, A.A. Starobinsky, Mon. Not. R. Astron. Soc. 344, 1057 (2003)

20. M. Arabsalmani, V. Sahni, Phys. Rev. D 83, 043501 (2011)

21. J. Li, R. Yang, B. Chen, JCAP 1412(12), 043 (2014)

22. I. Albarran, M. Bouhmadi-López, J. Morais, Phys. Dark Univ. 16, 94 (2017)

23. J. Väliviita, E. Majerotto, R. Maartens, JCAP 0807, 020 (2008)

24. R. Bean, O. Doré, Phys. Rev. D 69, 083503 (2004)

25. R. de Putter, D. Huterer, E.V. Linder, Phys. Rev. D 81, 103513 (2010)
26. G. Ballesteros, J. Lesgourgues, JCAP 1010, 014 (2010)

27. Y.S. Song, W.J. Percival, JCAP 0910, 004 (2009)

28. A .Y. Kamenshchik, E .O. Pozdeeva, S .Y. Vernov, A. Tronconi, G. Venturi, Phys. Rev. D 94(6), 063510 (2016)

29. V. Sahni, Y. Shtanov, JCAP 0311, 014 (2003)

30. S. Bag, A. Viznyuk, Y. Shtanov, V. Sahni, JCAP 1607(07), 038 (2016)

31. M .P. Dạbrowski, C. Kiefer, B. Sandhöfer, Phys. Rev. D 74, 044022 (2006)

32. A. Kamenshchik, C. Kiefer, B. Sandhöfer, Phys. Rev. D 76, 064032 (2007)

33. M. Bouhmadi-López, C. Kiefer, B. Sandhöfer, P. Vargas, Phys. Rev. D 79, 124035 (2009) 